The poster incorporated a check list on how to assess a neonate for signs of deterioration under the headings: Breathing, Colour, Temperature, Activity and Feeding. This was then taught to the nurses using clinical videos and bedside teaching in the neonatal unit. The nurses were asked to complete a test assessing their knowledge before and after the teaching session. The mean scores were compared using the student $\mathrm{t}$ test to determine if there was any statistically significant difference.

Result The data shows that there was a statistically significant improvement in the test scores after training in both hospitals. In Hospital $\mathrm{A}$, the average test score before training was $61.3 \%$ but improved to $81.3 \%$ after training $(p=0.007)$. In Hospital B the average test score before training was $83.3 \%$ and then $97.8 \%$ after training $(p=0.016)$.

Conclusion The training programme was effective in improving nursing knowledge in the recognition of the sick and deteriorating neonate as well as escalation of care.

\section{G467(P) ABSTRACT WITHDRAWN}

\section{British inherited metabolic disease group}

\section{G468 'SIMPLE BRONCHIOLITIS' - ARE YOU SURE?}

RS Tattersall, C Williams. Department of Child Health, Royal Cornwall Hospitals NHS Trust, Truro, UK

\subsection{6/archdischild-2020-rcpch.401}

Background A 7 month old boy was referred to paediatric admissions with 'simple bronchiolitis'. During his admission, however, mum mentioned that something was not right.

Case Presentation His bronchiolitis made feeding difficult and he tired easily. Admission was required for feeding support, with a nasogastric tube inserted.

Ward review noted that he had not met gross motor milestones and was hypotonic. He was unable to sit, even when supported, had some head lag and only a few anti-gravity movements. Faltering growth was demonstrated, dropping from the 91st to the 9th centile in the past few months. He was otherwise well, with a normal examination and no dysmorphic features.

Investigations were arranged, including a genetic and hypotonia screen. Prader-Willi and spinal muscular atrophy, in particular, were considered.

Bloods showed a raised ALT at 222 and CK at 908. After discussion with the metabolic team, further tests were requested, including an echocardiogram.

The echocardiogram showed septal and left ventricular hypertrophy with mild diastolic dysfunction. Pompe's disease was, therefore, considered. Alpha-glucosidase levels were checked, confirming this diagnosis.

$\mathrm{He}$ is now under follow-up at the tertiary centre, with multidisciplinary care locally, and has started life-long enzyme replacement therapy.

Pompe's disease is an autosomal recessive glycogen storage disorder that has variable rates of progression and time of onset. An earlier age of onset usually confers a worse prognosis. Mobility problems characterise the disease due to skeletal muscle weakness and subsequent wasting. Infantile onset Pompe's presents with hypertrophic cardiomyopathy, an enlarged liver from cardiac failure and respiratory problems due to wasting of the skeletal muscles.

Conclusion This case highlights the importance of ensuring a thorough history and examination for every child that comes to child health, even when the diagnosis seems clear and relatively benign. In the midst of the bronchiolitis season, doctors can be tempted to make assumptions to save time. However, ensuring each patient is reviewed properly enables rare diagnoses to be considered, confirmed and ensures children are started on the treatment they need.

Not just a case of 'simple bronchiolitis' after all!

\section{G469 DEVELOPMENTAL DELAY IN A YOUNG INFANT WITH NON-CLASSICAL COMBINED MALONIC AND METHYL MALONIC ACIDURIA (CMAMMA) CAUSED BY HOMOZYGOUS MISSENSE MUTATION IN ACSF3 GENE}

${ }^{1} \mathrm{MBKC}$ Dayasiri, ${ }^{1} \mathrm{E}$ Goh, ${ }^{1} \mathrm{~S}$ Kodagali, ${ }^{2} \mathrm{~J}$ Baruteau, ${ }^{1} \mathrm{G}$ Anand. ${ }^{1}$ Department of Paediatrics, Oxford University Hospital, Oxford, UK; ${ }^{2}$ Department of Paediatric Metabolic Medicine, Great Ormond Street Hospital, UK

\subsection{6/archdischild-2020-rcpch.402}

Introduction AcylCoA synthetase family member 3 (ACSF3) activates malonylCoA and methymalonylCoA into their respective thioesters. ACSF3 deficiency causes non-classical CMAMMA, a rare inborn error of metabolism characterised by presence of methyl malonic acid in higher concentrations than malonic acid in urine. The reversal is seen with classic CMAMMA caused by malonylCoA decarboxylase deficiency (MCD).

Case Report 5 and a half month old male infant second born to consanguineous South Asian parents presented with severe failure to thrive and recurrent vomiting. His older sibling who had failure to thrive and neuro-developmental delay died at seven months without a genetic diagnosis.

Initial blood tests revealed metabolic acidosis, pancytopenia and coagulopathy. Neuroimaging was unremarkable. Subsequent evaluation revealed normal levels of methionine, homocysteine and red cell folate. Significant methylmalonic aciduria with mild malonic aciduria without evidence of other abnormal metabolites (propionyl-CoA metabolites: hydroxypropionate, and methylcitrate or tiglylglycine) in urine suggested the diagnosis of non-classic CMAMMA, confirmed by homozygous missense variants in ACSF3 gene revealed by trio-exome sequencing. Neurodevelopmental assessment at eight months revealed global developmental delay with general hypotonia; gross motor (4-6 months); fine motor (4-6 months); speech (5-6 months) and social (under 6 months), and without any regression. Carnitine was supplemented to avoid secondary depletion caused by the excretion of MMA. Parents were advised to avoid prolonged fasting and to provide emergency regimen (powdered carbohydrate drink mix) in the event of acute deterioration.

Conclusion This report describes an unusual paediatric presentation of non-classic CMAMMA. Urine organic acids allows identification of increased MA and MMA excretion and highly suggestive of the diagnosis, thus avoiding additional investigations Determination of urinary $\mathrm{MMA} / \mathrm{MA}$ ratio can help differentiating between classical and non-classical forms. 